\title{
Tunable Klein-like tunnelling of high-temperature superconducting pairs into graphene
}

\author{
David Perconte ${ }^{1 \dagger}$, Fabian A. Cuellar ${ }^{1 \dagger}$, Constance Moreau-Luchaire', Maelis Piquemal-Banci', \\ Regina Galceran', Piran R. Kidambi ${ }^{2}$, Marie-Blandine Martin², Stephan Hofmann ${ }^{2}$, Rozenn Bernard', \\ Bruno Dlubak ${ }^{1}$, Pierre Seneor ${ }^{1 \star}$ and Javier E. Villegas ${ }^{1 \star}$
}

Superconductivity can be induced in a normal material via the 'leakage' of superconducting pairs of charge carriers from an adjacent superconductor. This so-called proximity effect is markedly influenced by graphene's unique electronic structure, both in fundamental and technologically relevant ways. These include an unconventional form ${ }^{1,2}$ of the 'leakage' mechanismthe Andreev reflection ${ }^{3}$-and the potential of supercurrent modulation through electrical gating ${ }^{4}$. Despite the interest of high-temperature superconductors in that context ${ }^{5,6}$, realizations have been exclusively based on low-temperature ones. Here we demonstrate a gate-tunable, high-temperature superconducting proximity effect in graphene. Notably, gating effects result from the perfect transmission of superconducting pairs across an energy barrier-a form of Klein tunnelling ${ }^{7,8}$, up to now observed only for non-superconducting carriers ${ }^{9,10}-$ and quantum interferences controlled by graphene doping. Interestingly, we find that this type of interference becomes dominant without the need of ultraclean graphene, in stark contrast to the case of low-temperature superconductors ${ }^{11}$. These results pave the way to a new class of tunable, high-temperature Josephson devices based on large-scale graphene.

Superconductivity is induced in a normal metal $(\mathrm{N})$ in contact with a superconductor $(S)$ via the Andreev reflection $(A R)^{3}$ : an electron entering $\mathrm{S}$ from $\mathrm{N}$ pairs to another electron to form a Cooper pair, leaving a hole-like quasiparticle that is transmitted back into N. Electron and hole coherently propagate with parallel opposite wavevectors, carrying superconducting correlations into N. This mechanism allows supercurrent flow and Josephson coupling across $\mathrm{S}-\mathrm{N}-\mathrm{S}$ junctions ${ }^{12}$.

S-N proximity devices that can be greatly tuned by electrostatic doping are one of the main technological prospects of induced superconductivity in graphene $e^{4,13-16}$. Several specific mechanisms allow for that. Besides the density-of-states narrowing at the Dirac point, around which the junction's resistance increases, subtler effects may play a role. For example, the unusual possibility that the AR-involved electron and hole reside in different bandsconduction and valence-results in a specular Andreev reflection ${ }^{1}$ (SAR) in which electron and hole wavevectors are mirror-like. SAR can occur if the graphene's Fermi energy $E_{\mathrm{F}}$ is lower than the superconducting energy gap $\Delta$, while for $E_{\mathrm{F}}>\Delta$ the conventional (intra-band) AR takes place ${ }^{1}$. Thus, an AR to SAR crossover can be driven by shifting $E_{\mathrm{F}}$ through a gate voltage, which dramatically changes the $\mathrm{S}-\mathrm{N}$ interface conductance ${ }^{2}$.
In the present experiments, tunability results from a different mechanism that involves Klein tunnelling-that is, the reflectionless transmission of electrons across a high energy barrier ${ }^{7-10}$. Beyond single electrons, here we observe Klein-like tunnelling of Andreev electron-hole pairs that carry superconducting correlations from the high-temperature superconductor $\mathrm{YBa}_{2} \mathrm{Cu}_{3} \mathrm{O}_{7}$ (YBCO) into graphene. That effect is associated with quantum interferences across the barrier, which modulate the overall penetration of superconducting pairs into graphene. Those interferences are gatetunable because the wavevector of graphene's massless charge carriers is proportional to $E_{\mathrm{F}}$. Although extremely sensitive to $E_{\mathrm{F}}$ inhomogeneities comparable to $\Delta$-which has confined their pristine observation to ultraclean exfoliated graphene ${ }^{11}$-we find that the large $\Delta$ of YBCO makes interference effects robust in chemical vapour deposited (CVD) graphene. This increases the technological potential of the observed effects.

Fabricating YBCO/graphene devices with electron-transparent interfaces has remained challenging ${ }^{17}$. Contrary to low-temperature superconductors ${ }^{2,4,11,13-16,18-20}$, YBCO cannot be grown on graphene due to its deposition conditions (hundreds of ${ }^{\circ} \mathrm{C}$, oxygen-rich atmosphere). Conversely, the surface electronic properties of YBCO are easily degraded by standard graphene fabrication and lithography techniques. To circumvent those constraints, we used 50-nm-thick YBCO films grown on $\mathrm{SrTiO}_{3}$ (STO) and covered in situ with an ultrathin $4 \mathrm{~nm}$ Au layer. This protects the YBCO surface and constitutes a high-transparency interface with graphene. Then we applied a masked ion irradiation technique (details in Fig. 1) that allowed us to fabricate planar devices as shown in Fig. 1e: four superconducting $\mathrm{YBCO} / \mathrm{Au}$ electrodes (bright colour) defined within an insulating YBCO matrix (dark colour) are bridged by an overlaying single-layer CVD graphene sheet. With this device, we study the $\mathrm{YBCO} / \mathrm{Au} /$ graphene junction conductance using a threeprobe configuration, with the current $I$ injected between electrodes 1 and 4 and the voltage $V$ measured between 3 and 4 (sketch in Fig. 1e). In that configuration the contributions of the YBCO leads and the $\mathrm{YBCO} / \mathrm{Au}$ interface to the measured conductance are negligible (see Supplementary Section 1). Thus, the measurement allows us to probe the Au/graphene conductance and, particularly, the proximity behaviour of that single interface. Notice also that the ultrathin Au layer is expected to sustain proximity-induced d-wave superconductivity (see Supplementary Section 1). We stress that here we do not investigate the Josephson effect across graphene, which is neither observed nor expected because the distance

${ }^{1}$ Unité Mixte de Physique, CNRS, Thales, Univ. Paris-Sud, Université Paris-Saclay, 91767 Palaiseau, France. ${ }^{2}$ Department of Engineering, University of Cambridge, Cambridge CB3 OFA, UK. `These authors contributed equally to this work. ${ }^{\star}$ e-mail: pierre.seneor@cnrs-thales.fr; javier.villegas@cnrs-thales.fr 
a

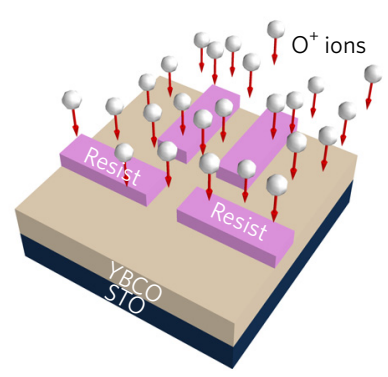

C
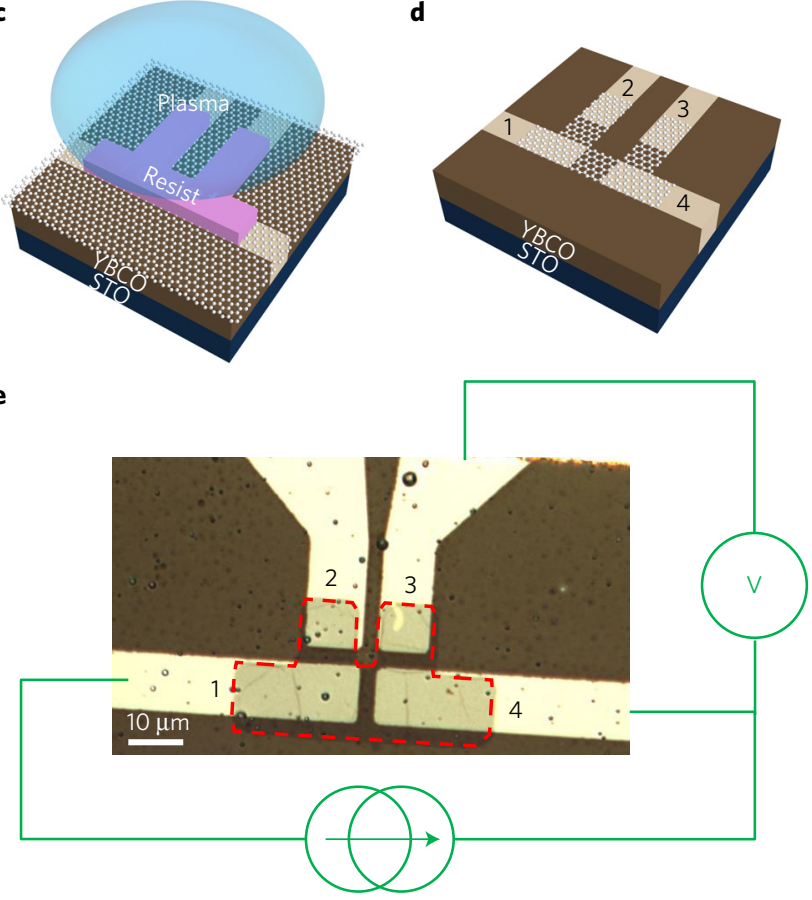

b

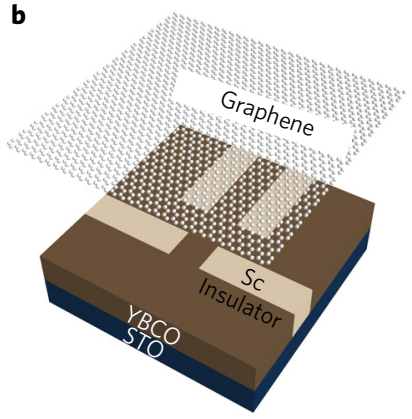

d

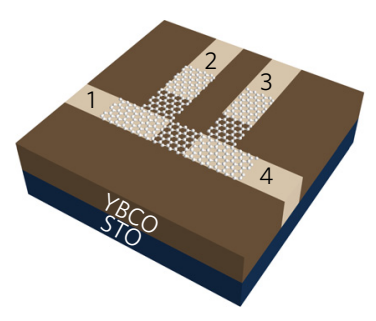

$V=60 \mathrm{mV}$ (this is well above the YBCO superconducting gap ${ }^{21}$ ). Magnetic fields up to $H=6 \mathrm{kOe}$ had no effect on $G(V)$ (data not shown). In contrast, $G(V)$ is strongly modulated by back-gating.

Following the scheme in Fig. $2 c$, the application of a gate voltage $V_{\mathrm{G}}$ leads to graphene doping due to the polarization of the substrate (STO) and the irradiated (insulating) YBCO. Fig. 2b illustrates the effects of $V_{\mathrm{G}}$. For convenience, the normalized conductance $g(V) \equiv G(V) / G_{\mathrm{N}}(V)$ is shown, with $G_{\mathrm{N}}(V)$ the normal-state one at $120 \mathrm{~K}$. The conductance under $V_{\mathrm{G}}=-40 \mathrm{~V}$ (bottom panel) is markedly different from that for $V_{\mathrm{G}}=0 \mathrm{~V}$ (top panel): changes in the zero-bias peak width and height, in the dips' depth and in the conductance background are observed. Notice, for example, a conductance enhancement $(g>1)$ for $V_{\mathrm{G}}=-40 \mathrm{~V}$ (bottom) within the bias range in which dips and $g<1$ are observed for $V_{\mathrm{G}}=0 \mathrm{~V}$ (top). As discussed below, the curves in Fig. $2 \mathrm{~b}$ constitute two behaviour types between which $g(V)$ periodically switches as a function of $V_{\mathrm{G}}$.

Figure 3a displays $g\left(V, V_{\mathrm{G}}\right)$ in a contour plot generated from a series of measurements such as those in Fig. 2b (data range is $|V|<30 \mathrm{mV}$ (around 1.5 $\Delta$ ) and $\left|V_{\mathrm{G}}\right|<100 \mathrm{~V}$ in this figure; the data for the whole experimental window is displayed in Supplementary Fig. 6). The zero-bias peak observed for all $V_{\mathrm{G}}$ (Fig. 2b) appears in the contour plot as a vertical 'band' (in red). The conductance dips observed around $\pm \sim 20 \mathrm{mV}$ in Fig. $2 \mathrm{~b}$ show up in the plot as 'pockets' (in purple colour). The background enhancement $(g>1)$ seen in Fig. 2b for $V_{\mathrm{G}}=-40 \mathrm{~V}$ and $|V|<40 \mathrm{mV}$ is shown in the plot as a horizontal feature (in green). Purple 'pockets' and green horizontal features alternate periodically along the $y$-axis-that is, as a function of $V_{\mathrm{G}}$. This periodic modulation is highlighted in Fig. 3b, which displays $g\left(V_{\mathrm{G}}\right)$ for fixed $V$-this corresponds to vertical 'cuts' of the contour plot, marked with the dashed lines $\Theta$ and $\Sigma$ in Fig. 3a. Notice (Fig. 3b) that the periodic modulation is accompanied by a conductance background decrease for increasing $V_{\mathrm{G}}$. Further analysis of the oscillatory behaviour can be found in Supplementary Fig. 7.

To interpret the experimental results, we start by considering the conductance across generic superconductor/normal (S/N) interfaces $^{22}$. In this context, the zero-bias conductance doubling at low $T$ (Fig. 2a) suggests a highly transparent $\mathrm{S} / \mathrm{N}$ interface governed by $\mathrm{AR}^{22}$ (devices showing reduced transparency are discussed in Supplementary Section 2). The invariance of the conductance curves with respect to the magnetic field is consistent with that scenario. $V_{\mathrm{G}}$ effects imply that graphene is necessarily the ' $\mathrm{N}$ ' part of the $\mathrm{S} / \mathrm{N}$ junction, and rule out a possible role of the $\mathrm{YBCO} / \mathrm{Au}$ interface ${ }^{23}$. This is because the device geometry ensures that $V_{\mathrm{G}}$ solely affects graphene that lies on insulating YBCO (dark in Fig. 2c). The electric field is fully screened elsewhere by the superconducting YBCO (bright in Fig. 2c), whose properties are unaffected by $V_{\mathrm{G}}$ because its thickness is $\sim 50$ times the Thomas-Fermi screening length ${ }^{24}$.

The overall experimental details are explained by the model sketched in Fig. 3g. This model is based on the expectation that graphene on top of superconducting $\mathrm{YBCO} / \mathrm{Au}$ becomes superconducting $(\mathrm{S})$ due to the proximity effect ${ }^{1}$ and presents a fixed doping $E_{\mathrm{F}}^{\prime}$, while graphene on top of insulating YBCO is normal $(\mathrm{N})$ and presents a gate-tunable doping $E_{\mathrm{F}}\left(V_{\mathrm{G}}\right)$. Then we assume that there is an intermediate region $\mathrm{N}^{\prime}$ of width $w$ in which graphene is normal but presents different doping than in S and N (see Supplementary Section 5). This scenario leads to a potential energy step $U_{0}$ for electrons at the boundary between $\mathrm{S}$ and $\mathrm{N}$ graphene ${ }^{6}$ (note that replacing $\mathrm{S}$ by normal graphene $(\mathrm{N})$ results in a type of graphene $\mathrm{N}-\mathrm{N}^{\prime}-\mathrm{N}$ junction in which regular-non-superconducting-Klein tunnelling is expected ${ }^{7-10}$ ). The conductance across such an $\mathrm{S}-\mathrm{N}^{\prime}-\mathrm{N}$ junction has been theoretically studied both for $s$-wave $e^{5,6,25}$ and d-wave superconductors ${ }^{5,6}$ (note that in those works exactly the same junction structure was referred to as S-I-N). The central effect arises due to interferences of electrons and holes travelling from the 

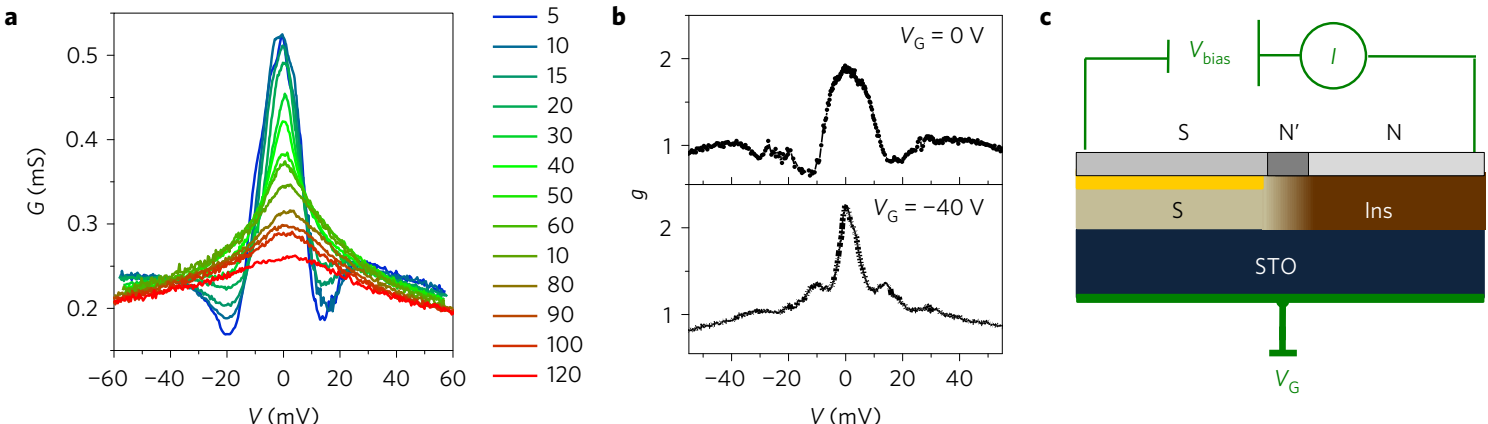

Figure 2 | Temperature and gate-voltage dependent conductance. a, Differential conductance of the YBCO/Au/graphene junction in zero applied field and different temperatures (in K). b, Normalized conductance at zero magnetic field and $T=4 \mathrm{~K}$ for two gate voltages: $V_{\mathrm{G}}=0 \mathrm{~V}$ (top) and $V_{\mathrm{G}}=-40 \mathrm{~V}$ (bottom). c, Scheme of the device cross-section. Graphene, in grey, is superconducting (S) above the superconducting YBCO (light colour), and normal ( ${ }^{\prime}$ and N) elsewhere. The insulating (Ins) YBCO is depicted in dark colour. The boundary between superconducting and insulating YBCO has a width of a few tens of nanometres (graded colour). Gating is achieved across the dielectric STO substrate.
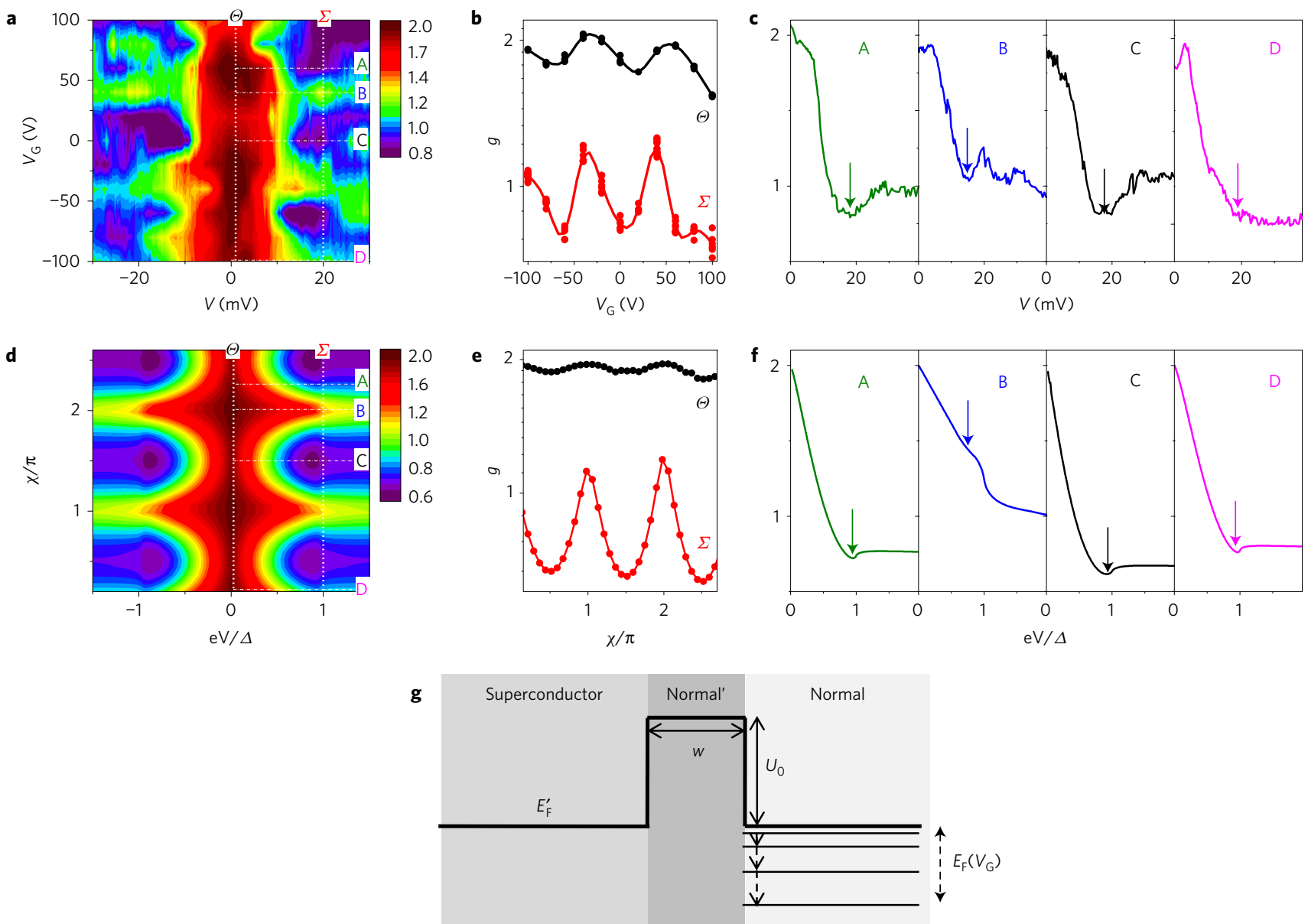

Figure 3 | Gate-voltage effects: experiment and simulations. a, Contour plot of the normalized conductance (colour scale) as a function of junction bias $V$ and gate voltage $V_{G}$. This plot is constructed from a series of measurements as those shown in Fig. 2 , with $\Delta V_{G}=20$ V. $\mathbf{b}$, Experimental conductance as a function of gate voltage $V_{\mathrm{G}}$ at fixed bias $V$, which correspond to vertical 'cuts' of the plot in a along the dashed lines labelled $\Theta$ and $\Sigma$ (respectively for $V=0 \pm 1 \mathrm{mV}$ and $V=20 \pm 1 \mathrm{mV}$ ). Various points per $V_{G}$ exists which denote the measurement uncertainty (see Supplementary Section 7 for further details). The solid line behind the data is a guide to the eye. $c$, Experimental conductance versus bias $V$ for fixed gate voltages $V_{G}$. Each panel corresponds to a horizontal 'cut' of the plot in a along the lines labelled $A, B, C, D$ (respectively for $V_{G}=60,40,0$ and $-100 \mathrm{~V}$ ). The arrow points to the conductance dip below $\mathrm{eV}=\Delta \mathbf{.} \mathbf{d}-\mathbf{f}$, Numerical simulations corresponding to the measurements in $\mathbf{a}-\mathbf{c}$. Notice that in the simulation the bias $V$ is normalized by the superconducting gap $\Delta$, and the experimental quantity $V_{G}$ is replaced by the phase $\chi \cdot \mathbf{g}$, Schematic of the theoretical model. A potential step of width $w$ and height $U_{0}$ defines a region of normal graphene $\left(N^{\prime}\right)$ that separates superconducting graphene with Fermi energy $E_{F}^{\prime}$ from normal graphene $(N)$ with gate-tunable Fermi energy $E_{F}\left(V_{G}\right)$.

$\mathrm{N}^{\prime}-\mathrm{N}$ to the $\mathrm{S}-\mathrm{N}^{\prime}$ interface (and vice versa after reflection at the $\mathrm{S}-\mathrm{N}^{\prime}$ interface). Depending on the phase $\chi=-w k_{\mathrm{F}}$ picked up by electrons/holes across $\mathrm{N}^{\prime}$ (with $k_{\mathrm{F}}$ the Fermi vector), constructive or destructive interference occurs, which periodically modulates the junction conductance as a function of $\chi^{6}$. Thus, due to graphene's linear dispersion, $\chi=w\left(E_{\mathrm{F}}-U_{0}\right) / \hbar v_{\mathrm{F}}$ (with $v_{\mathrm{F}}$ the Fermi velocity) 
and a periodic conductance modulation as a function of $E_{\mathrm{F}}$ is expected. That can explain the gating effects observed in Fig. 3a,b. To support this, we used the existing theory ${ }^{6}$ to perform the numerical simulations shown in Fig. 3d-f.

Simulations of $g(\mathrm{eV} / \Delta, \chi)$ require several input parameters: the native Fermi energy in $N, E_{\mathrm{F}}(0)$; the Fermi energy in $S, E_{\mathrm{F}}^{\prime}$; the proportionality factor between $E_{\mathrm{F}}$ variation and phase shift $\delta E_{\mathrm{F}} / \delta \chi$; and the angle $\alpha$ between the d-wave nodes and the S- $\mathrm{N}^{\prime}$ interface ${ }^{5}$. As discussed in Supplementary Section 4, most of these parameters are known from independent experiments, and only $\alpha$ and $E_{\mathrm{F}}$ remain as fitting parameters. Figure $3 \mathrm{~d}$ shows the best agreement between theory and experiments, obtained for $\alpha=\pi / 4$, $E_{\mathrm{F}}^{\prime}=20 \Delta, E_{\mathrm{F}}(0)=17.5 \Delta$, and $\delta E_{\mathrm{F}} / \delta \chi=5 \Delta / 3 \pi$. Notably, all of the characteristic features seen in the experimental $g\left(V, V_{\mathrm{G}}\right)$ (Fig. 3a) can be found in Fig. 3d: a vertical band (in red) that corresponds to a zero-bias conductance peak due to AR, and a periodic modulation along the $y$-axis in which horizontal features due to enhanced conductance (in green) alternate with 'pockets' (in purple). Figure $3 \mathrm{e}$ shows vertical 'cuts' of the theoretical plot along the lines $\Theta$ and $\Sigma$, which closely mimic the experimental ones (Fig. 3b). 'Cuts' along the $x$-axis (labelled A, B, C, D) are also shown for experiments and theory, respectively, in Fig. 3c,f. Their comparison show that the simulations not only successfully reproduce the periodic gating effects, but also the essential details of the experimental $g(V)$ : a conductance enhancement around zero bias, 'dips' around $\mathrm{eV} \sim \Delta$, and the evolution of these features with $V_{\mathrm{G}}$ (phase $\chi$ ). The overall agreement between simulation and experiment demonstrates that the used model $^{6}$ captures the physics of the studied system. Further improving the match between theory and experiment, particularly for $\mathrm{eV}>\sim 1.5 \Delta$ where discrepancies appear (see Supplementary Fig. 6), may require refining the model, for example, by considering different barrier profiles (for example, trapezoidal instead of square), smeared or rough $\mathrm{S} / \mathrm{N}^{\prime} / \mathrm{N}$ interfaces, and so on (see discussion in Supplementary Section 5). We hope that the present experiments will motivate that work.

In summary, the periodic modulation of the conductance is explained by electron interferences within an energy barrier that separates normal and superconducting graphene. The barrier is essentially transparent to electron/hole superconducting pairs (as well as for normal electrons) when the phase picked across the barrier is $\chi=n \pi$, which corresponds to Klein tunnelling of superconducting pairs. Thus, the interferences allow for an electrostatic tuning of the superconducting proximity effect, since the fraction of the current carried by electron-hole superconducting pairs (created by AR) is modulated by $\chi\left(V_{\mathrm{G}}\right)$ (ref. 6). These results open interesting perspectives. For instance, experiments aimed at selecting the pairing symmetry induced in graphene via the control of the orientation of the graphene and YBCO lattices ${ }^{26}$, gatetunable high-temperature Josephson devices using large-scalenot necessarily ultraclean-graphene, and possibly more elaborate devices in which those effects, the salient features of d-wave superconductivity and the directional nature of Klein tunnelling ${ }^{7}$ may be exploited together, for example, to create tunable 'pi' Josephson junctions ${ }^{27}$.

Note added in proof: During the resubmission of the paper we learnt about the publication of related scanning tunnelling microscopy experiments of graphene on a different cuprate superconductor ${ }^{28}$.

\section{Methods}

Methods, including statements of data availability and any associated accession codes and references, are available in the online version of this paper.

Received 26 April 2017; accepted 30 August 2017; published online 9 October 2017

\section{References}

1. Beenakker, C. W. J. Specular Andreev reflection in graphene. Phys. Rev. Lett. 97, 67007 (2006).

2. Efetov, D. K. et al. Specular interband Andreev reflections at van der Waals interfaces between graphene and $\mathrm{NbSe}_{2}$. Nat. Phys. 12, 328-332 (2015),

3. Andreev, A. F. The thermal conductivity of the intermediate state in superconductors. Sov. Phys. JETP 19, 1228-1231 (1964).

4. Heersche, H. B., Jarillo-Herrero, P., Oostinga, J. B., Vandersypen, L. M. K. \& Morpurgo, A. F. Bipolar supercurrent in graphene. Nature 446, 56-59 (2007).

5. Linder, J. \& Sudbø, A. Dirac fermions and conductance oscillations in s- and d-wave superconductor-graphene junctions. Phys. Rev. Lett. 99, 147001 (2007)

6. Linder, J. \& Sudbø, A. Tunneling conductance in s- and d-wave superconductor-graphene junctions: extended Blonder-Tinkham-Klapwijk formalism. Phys. Rev. B 77, 64507 (2008).

7. Katsnelson, M., Novoselov, K. \& Geim, A. Chiral tunnelling and the Klein paradox in graphene. Nat. Phys. 2, 620-625 (2006)

8. Beenakker, C. W. J. Colloquium: Andreev reflection and Klein tunneling in graphene. Rev. Mod. Phys. 80, 1337-1354 (2008).

9. Huard, B. et al. Transport measurements across a tunable potential barrier in graphene. Phys. Rev. Lett. 98, 236803 (2007).

10. Young, A. F. \& Kim, P. Quantum interference and Klein tunnelling in graphene heterojunctions. Nat. Phys. 5, 222-226 (2009).

11. Ben Shalom, M. et al. Quantum oscillations of the critical current and high-field superconducting proximity in ballistic graphene. Nat. Phys. 12, 318-322 (2015)

12. Klapwijk, T. M. Proximity effect from an Andreev perspective. J. Supercond. 17, 593-611 (2004)

13. Du, X., Skachko, I. \& Andrei, E. Y. Josephson current and multiple Andreev reflections in graphene SNS junctions. Phys. Rev. B 77, 184507 (2008)

14. Ojeda-Aristizabal, C., Ferrier, M., Guéron, S. \& Bouchiat, H. Tuning the proximity effect in a superconductor-graphene-superconductor junction. Phys. Rev. B 79, 165436 (2009).

15. Girit, C.. et al. Tunable graphene dc superconducting quantum interference device. Nano Lett. 9, 198-199 (2009).

16. Komatsu, K., Li, C., Autier-Laurent, S., Bouchiat, H. \& Guéron, S. Superconducting proximity effect in long superconductor/graphene/ superconductor junctions: from specular Andreev reflection at zero field to the quantum Hall regime. Phys. Rev. B 86, 115412 (2012).

17. Sun, Q. J. et al. Electronic transport transition at graphene $/ \mathrm{YBa}_{2} \mathrm{Cu}_{3} \mathrm{O}_{7}-\delta$ junction. Appl. Phys. Lett. 104, 102602 (2014).

18. Rickhaus, P., Weiss, M., Marot, L. \& Schönenberger, C. Quantum Hall effect in graphene with superconducting electrodes. Nano Lett. 12, 1942-1945 (2012).

19. Deon, F., Šopić, S. \& Morpurgo, A. F. Tuning the influence of microscopic decoherence on the superconducting proximity effect in a graphene Andreev interferometer. Phys. Rev. Lett. 112, 126803 (2013).

20. Allen, M. T. et al. Spatially resolved edge currents and guided-wave electronic states in graphene. Nat. Phys. 12, 128-133 (2016).

21. Wei, J. Y. T., Yeh, N.-C., Garrigus, D. F. \& Strasik, M. Directional tunneling and Andreev reflection on $\mathrm{YBa}_{2} \mathrm{Cu}_{3} \mathrm{O}_{7-\delta}$ single crystals: predominance of d-wave pairing symmetry verified with the generalized Blonder, Tinkham, and Klapwijk theory. Phys. Rev. Lett. 81, 2542-2545 (1998).

22. Blonder, G. E., Tinkham, M. \& Klapwijk, T. M. Transition from metallic to tunneling regimes in superconducting micro-constrictions-excess current, charge imbalance, and super-current conversion. Phys. Rev. B 25, 4515-4532 (1982)

23. Kashiwaya, S., Tanaka, Y., Koyanagi, M., Takashima, H. \& Kajimura, K. Origin of zero-bias conductance peaks in high- $T_{\mathrm{c}}$ superconductors. Phys. Rev. B 51, $1350-1353$ (1995)

24. Crassous, A. et al. Nanoscale electrostatic manipulation of magnetic flux quanta in ferroelectric/superconductor $\mathrm{BiFeO}_{3} / \mathrm{YBa}_{2} \mathrm{Cu}_{3} \mathrm{O}_{7-\delta}$ heterostructures. Phys. Rev. Lett. 107, 247002 (2011).

25. Bhattacharjee, S. \& Sengupta, K. Tunneling conductance of graphene NIS junctions. Phys. Rev. Lett. 97, 217001 (2006).

26. Linder, J., Black-Schaffer, A. M., Yokoyama, T., Doniach, S. \& Sudbø, A Josephson current in graphene: role of unconventional pairing symmetries. Phys. Rev. B 80, 94522 (2009).

27. Cedergren, K. et al. Interplay between static and dynamic properties of semifluxons in $\mathrm{YBa}_{2} \mathrm{Cu}_{3} \mathrm{O}_{7-\delta} 0-\pi$ Josephson junctions. Phys. Rev. Lett. 104, 177003 (2010)

28. Bernard, A. Di et al. p-wave triggered superconductivity in single-layer graphene on an electron-doped oxide superconductor. Nat. Commun. 8 , 14024 (2017). 


\section{Acknowledgements}

Work at CNRS/Thales was supported by the French National Research Agency through 'Investissements d'Avenir' program Labex NanoSaclay (ANR-10-LABX-0035) and by the EU Work Programme under Grant Graphene Flagship (No. 604391) and Core1 (No. 696656). R.G. acknowledges funding from the Marie-Curie-ITN

607904-SPINOGRAPH. S.H. acknowledges funding from EPSRC grants EP/K016636/1 and EP/P005152/1. P.S. acknowledges the Institut Universitaire de France for a junior fellowship. We thank A. S. Mel'Nikov, J. Linder, J. Santamaría, S. Gueron and H. Bouchiat for useful discussions. We thank Y. Le Gall for assistance during ion irradiation.

\section{Author contributions}

J.E.V. and P.S. conceived the experiments. R.B. fabricated the YBCO films. P.R.K., M.-B.M. and S.H. fabricated the graphene sheets. F.A.C., fabricated the devices, with contributions from D.P., B.D., R.G. and M.P.-B. F.A.C. performed transport experiments, with contributions of D.P. and C.M.-L. D.P. performed numerical simulations. The figures were prepared and the paper written by D.P., F.A.C. and J.E.V., with contributions from all the other authors. All of the authors participated in the discussion of the results.

\section{Additional information}

Supplementary information is available in the online version of the paper. Reprints and permissions information is available online at www.nature.com/reprints. Publisher's note: Springer Nature remains neutral with regard to jurisdictional claims in published maps and institutional affiliations. Correspondence and requests for materials should be addressed to P.S. or J.E.V.

\section{Competing financial interests}

The authors declare no competing financial interests. 


\section{Methods}

$c$-axis YBCO thin films (50 nm thick) were grown on (100) $\mathrm{SrTiO}_{3}(\mathrm{STO})$ substrates $\left(500 \mu \mathrm{m}\right.$ thick) by pulsed-laser deposition at $700{ }^{\circ} \mathrm{C}$ and $0.35 \mathrm{mbar}$ of pure oxygen. The optimum oxygen stoichiometry is ensured by cooling down to room temperature in $800 \mathrm{mbar}$ of pure oxygen. Subsequently the chamber base pressure $\left(3 \times 10^{-6}\right.$ torr $)$ is reinstated, and an ultrathin Au film $(\sim 4 \mathrm{~nm}$ thick $)$ is deposited in situ on top of the YBCO to protect its surface. YBCO $c$-axis epitaxial growth is confirmed by reflection high-energy electron diffraction (in situ) and X-ray diffraction (ex situ), and film thicknesses are determined by $\mathrm{X}$-ray reflectivity. Atomic force microscopy of the Au/YBCO bilayer shows flat surfaces with typical r.m.s. roughness $\sim 3 \mathrm{~nm}$.

Graphene transfer onto the lithographed devices was done as reported elsewhere ${ }^{29}$.
Electrical characterization within the $3 \mathrm{~K}-300 \mathrm{~K}$ range was carried in a He-flow cryostat equipped with a $6 \mathrm{kOe}$ electromagnet. In the three-probe configuration, the current is injected between contacts 1 and 4 , and the voltage probes are attached to contacts 3 and 4 (see image in Fig. 1e). $\mathrm{d} I / \mathrm{d} V(V)$ is obtained using the current-biased Keithley delta mode with a coupled K2182 nanovoltmeter and K6221 current source.

Data availability. The data supporting the plots within this paper and other findings of this study are available from the corresponding author on reasonable request.

\section{References}

29. Kidambi, P. R. et al. The parameter space of graphene CVD on polycrystalline Cu. J. Phys. Chem. C 116, 22492-22501 (2012). 